\title{
Original
}

\section{Manejo laparoscópico de las anomalias sintomáticas del uraco}

\author{
Alexis Sánchez-Ismayel, Germán Cruz-González*, Renata Sánchez, Rodolfo Sánchez-Salas*, \\ Omaira Rodríguez, Erasmo Sanabria, Rene Sotelo**, Rafael E. Sánchez-Salas*
}

\begin{abstract}
Servicio de Cirugía III. *Servicio de Urología. Hospital Universitario de Caracas, Universidad Central de Venezuela. **Centro de Cirugía Robótica y Minimamente invasiva. Instituto Médico La Floresta. Caracas, Venezuela
\end{abstract}

\begin{abstract}
Resumen
Introducción: Las anomalías uracales son una patología poco frecuente, el tratamiento aceptado consiste en la resección del uraco en todo su trayecto con o sin resección parcial de la vejiga. Nuestro objetivo es actualizar nuestra experiencia en el tratamiento de anomalías sintomáticas del uraco mediante cirugía de mínimo acceso.

Pacientes y Métodos: Entre los años 2001 y 2007 se operaron 14 pacientes con anomalías adquiridas sintomáticas del uraco en nuestra institución. Se utilizó una técnica de tres portales previamente descrita por los autores, mediante la cual se realiza abordaje de la cavidad abdominal, confirmación del diagnóstico clínico inicial y resección del uraco en todo su trayecto en sentido cráneo caudal hasta la vejiga. Los datos demográficos y operatorios fueron recolectados de manera prospectiva y el análisis se realizó retrospectivamente.

Resultados: El tiempo quirúrgico promedio fue de 63 minutos $(45,110)$ con sangrado mínimo, sin casos de conversión y sin complicaciones asociadas a la técnica. La gran mayoría de los pacientes egresó durante las primeras 24 horas. En un tiempo promedio de seguimiento de 22 meses no se han reportado recurrencias de la patología uracal.

Conclusión: La laparoscopia juega un importante papel en el manejo de las anomalías sintomáticas del uraco, ya que permite confirmar el diagnóstico clínico y realizar la resección del uraco de manera segura y eficaz, aportando las ya conocidas ventajas de la cirugía de mínimo acceso. Los estudios de evaluación preoperatoria son de poca utilidad en la confirmación de esta patología.
\end{abstract}

Palabras clave: Anomalía del uraco. Tratamiento. Laparoscopia.

\section{Laparoscopic management of symptomatic urachal anomalies}

\section{Abstract}

Introduction: Acquired urachal anomalies are a rare pathology. Gold-standard treatment for this clinical situation remains the resection of the urachus in its entire tract with or without partial resection of the bladder. Our aim is to up-date authors's experience in the minimally invasive surgical treatment of acquired urachal disease.

Methods: From 2001 to 2007, 14 patients were operated for acquired urachal disease at our institution. A three portal technique previously described by the authors was employed. The diagnosis of acquired uracal disease was confirmed in all cases and the resection of the urachus in its entire tract performed in cephalocaudal direction onto the bladder. Operative and demographic data was prospectively collected and analysis retrospectively performed.

Results: Mean operative time was 63 minutes $(45,110)$, minimal blood loss, and no conversions to open surgery or perioperative complications were verified. The majority of the patients were discharged in the first 24 hours. At a follow-up of 22 months no recurrences of urachal pathology recurrences have been verified.

Conclusions: Laparoscopy plays a significant role in the management of symptomatic urachus anomalies. It allows objective confirmation of clinical diagnosis and adequate resection of the urachus in a safe and efficient fashion, while providing the well-known advantages of minimally acces surgery. Preoperative evaluation work-up has minimal impact of therapeutical decision.

Keywords: Urachal anomalies. Treatment. Laparoscopy.

$\mathrm{L}^{2}$ as anomalías del uraco no son frecuentes, se calcula en 1 en 5000 y además no todas llegarán a manifestarse clínicamente ${ }^{1}$, sin embargo, el cirujano no debe olvidar esta patología cuando evalúa pacientes con episodios de secreción umbilical o inflamación periumbilical. El uraco es el remanente embrionario de la alantoides, esta estructura aparece tempranamente en el desarrollo fetal, alrededor del decimosexto día, derivada del endodermo. Hacia el quinto mes de gestación la alantoides sufre una 
degeneración hasta obliterarse completamente su luz, eliminándose, de esta manera, la comunicación entre la vejiga ya formada y el ombligo, dando origen al uraco, el cual se localiza en el tejido conjuntivo laxo entre la fascia transversalis y el peritoneo; su parte terminal cursa intramuralmente en la pared vesical $^{2}$.

El primer caso de anomalía sintomática del uraco conocido fue descrito en un joven de 18 años de edad, identificado y tratado por Bartholomaeus Cabrolius en 1550, posteriormete Begg en 1927 recopila de la literatura a su disposición un total de 58 casos $^{3}$. Las anomalías del uraco no son una patología común y la literatura se basa fundamentalmente en reporte de casos aislados y muy cortas series.

La mayoría de las anomalías se diagnostican clínicamente al evidenciar la salida de secreción acuosa a través de la cicatriz umbilical, tal y como lo describieron Sterling y Goldsmith ${ }^{4}$. Los estudios imagenológicos usualmente utilizados son el ecosonograma abdominal, sinografía, cistografía e incluso la Tomografía abdominal computarizada y la Resonancia magnética nuclear ${ }^{1,5}$.

El tratamiento tradicional de las anomalías del uraco consiste en la resección del uraco desde el ombligo hasta la vejiga, con o sin resección parcial de esta última, a través de una incisión media infraumbilical o suprapúbica transversa ${ }^{6}$. La resección en todo el trayecto es lo aceptado, ya que disminuye la tasa de recurrencia, que puede llegar al $30 \%{ }^{7}$, a la vez que evita la posibilidad de degeneración maligna en segmentos no resecados $^{8}$.

Luego del primer reporte de abordaje laparoscópico de las anomalías del uraco en $1993^{9}$, numerosos casos aislados han sido publicados y seis cortas series todas con resultados similares.

Los autores describieron una técnica laparoscópica con el uso de tres portales ${ }^{10}$, luego de lo cual se han acumulado catorce casos en un lapso de seis años de estudio y seguimiento, lo que viene a ser una de las series más grande a nivel mundial.

\section{MATERIAL Y MÉTODOS}

Se trata de un estudio prospectivo, longitudinal, descriptivo, donde se estudiaron pacientes que asistieron a consulta en los servicio de cirugía o urología del Hospital Universitario de Caracas, con clínica sugestiva de anomalía del uraco, en el período comprendido entre junio 2001 y junio 2007.
El estudio de estos pacientes incluye, realización de historia clínica, y estudios complementarios:

1. Laboratorio preoperatorio, 2. ultrasonido abdominal con énfasis en pared abdominal en trayecto entre cicatriz umbilical y vejiga, 3. cistografía y 4. fistulografía o sinograma, en los casos en que se evidenció un trayecto permeable y fue posible su cateterización.

El único criterio de exclusión lo constituyo la contraindicación de realizar un procedimiento laparoscópico debido a patología médica asociada.

Una vez confirmado el diagnóstico, previa firma de consentimiento informado, se llevo a cabo la intervención quirúrgica laparoscópica según la técnica propuesta.

\section{Descripción de la Técnica}

Baja anestesia general, el paciente se coloca en decúbito dorsal, con Trendelenburg $30^{\circ}$, ligeramente lateralizado hacia la izquierda, para lo cual puede colocarse un soporte acolchado en la espalda del paciente. El cirujano y el asistente de cámara se colocan del lado derecho del paciente y el monitor del lado contralateral.

Se requiere de una torre de laparoscopia completa con insuflador autorregulable de alta presión, fuente de luz, cámara y monitor. Es indispensable el uso de una óptica de 30 ó 45 grados para lograr una óptima visión de la pared abdominal anterior.

El instrumental para la realización del procedimiento, consta de trocares de $10 \mathrm{~mm}$, disectores, pinzas de prehensión, tijeras y pinzas para colocación de clips, opcionalmente puede utilizarse el bisturí armónico. Entre otros accesorios debe contarse con una sonda de Foley y el equipo necesario para instilar solución en la vejiga.

Se utilizó una técnica de tres portales. El primer trocar se coloca por técnica abierta de Hasson, a nivel paraumbilical derecho, por fuera del borde externo del recto abdominal, posterior a lo cual se realiza la laparoscopia diagnóstica y se procede a la colocación de los demás portales; el segundo de ellos en el epigastrio, siempre bajo visión directa del laparoscopio, evitando de esta manera el ligamento redondo; y el tercero hacia la fosa ilíaca derecha, también por fuera del borde externo del recto con especial atención en evitar los vasos epigástricos.

La cámara se introduce a través del portal epigástrico, es indispensable el uso de una óptica de 30 grados para visualizar adecuadamente la pared 
abdominal, una vez identificada la variante anatómica y la anomalía del uraco, el cirujano utiliza los portales laterales para la disección del uraco en todo su trayecto, con el uso de pinzas de prehensión, disectores y tijeras (Fig. 1). El uso del bisturí armónico facilita la disección, contribuye con la hemostasia, a la vez disminuye el tiempo quirúrgico.

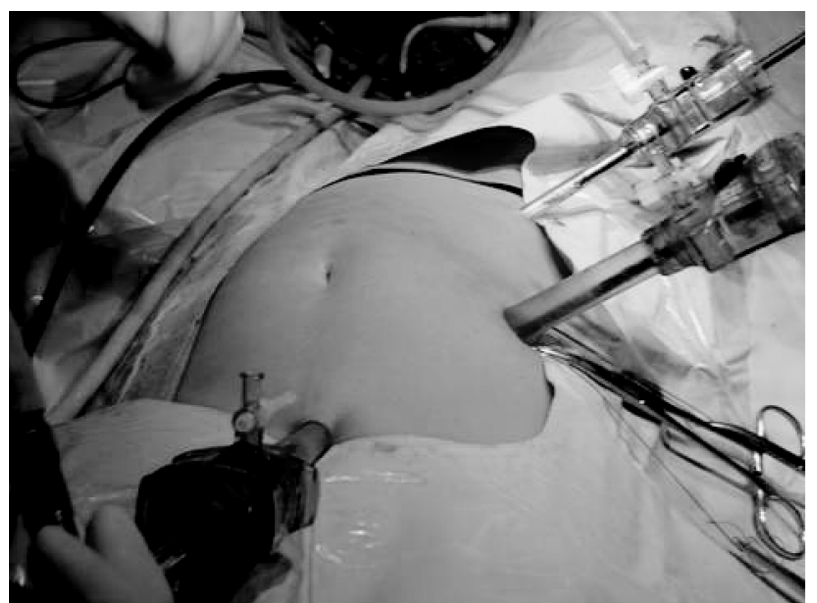

FIGURA 1. Ubicación de los portales. El cirujano trabaja con los portales laterales en dirección cráneo caudal.

Una vez identificado el nivel del extremo umbilical, se procede a la apertura del peritoneo parietal, identificación y disección del uraco en sentido cráneo caudal hasta la vejiga, la cual ha sido llenada previamente con 350cc de solución fisiológica teñida con azul de metileno para facilitar la identificación del extremo vesical. En ambos extremos del uraco deben colocarse clips y en ocasiones, dependiendo de la variedad anatómica que se encuentre, es necesario colocar clips y seccionar las arterias umbilicales.

No se consideró la resección parcial de la vejiga debido al muy bajo riesgo de malignización de la porción intravesical y la mayor morbilidad y tiempo quirúrgico asociado con la cistectomía parcial.

Finalmente la pieza es extraída a través de uno de los portales y enviada a estudio anatomopatológico (Fig. 2), se revisa de nuevo la cavidad abdominal y se extraen los trocares bajo visión directa. Posteriormente se procede al cierre de los portales que debe incluir el cierre del plano aponeurótico con sutura absorbible y síntesis de la piel.

\section{RESULTADOS}

Se estudiaron un total de doce pacientes de sexo femenino y dos pacientes masculinos, con una edad

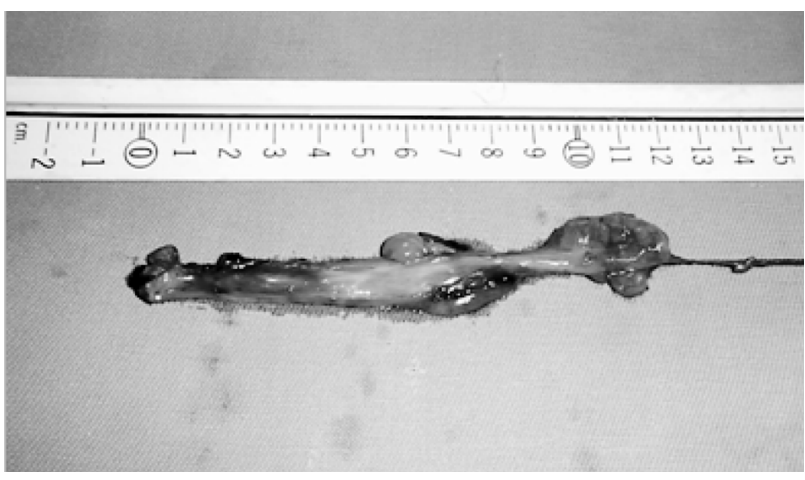

FIGURA 2. Especimen enviado para estudio histopatológico.

promedio de 19 años (rango 15 - 37). El diagnóstico de anomalía uracal se realizó en base a la clínica, caracterizada por episodios de secreción umbilical acuosa e inflamación periumbilical. Uno de los casos fue diagnosticado durante la realización de procedimiento laparoscópico por apendicitis aguda y posteriormente referido a nuestro centro.

Los estudios complementarios fueron de poca utilidad, sólo en un caso se logró la identificación de una imagen anecoica en línea media infraabdominal correspondiente con una lesión de uraco, durante el estudio ultrasonográfico. La cateterización del trayecto del uraco fue posible únicamente en un paciente, evidenciándose seno uracal en extremo umbilical. No se planteó la realización de tomografía abdominal computarizada de rutina debido a su alto costo y bajo disponibilidad.

La técnica propuesta permite visualizar el uraco en todo su trayecto para confirmación del diagnóstico y disección completa del mismo desde su extremo umbilical hasta el vesical (Fig. 3). En el último

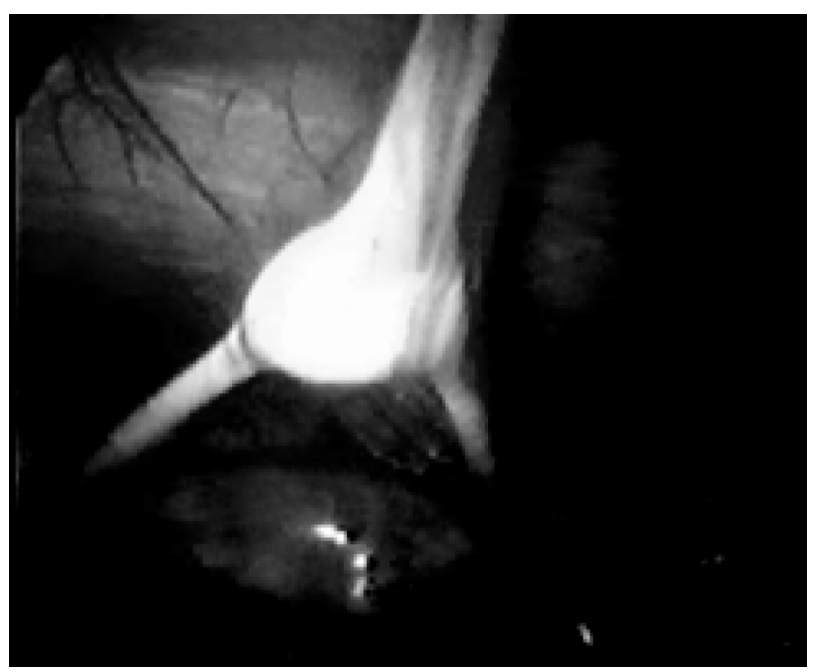

FIGURA 3. Visión laparoscópica de anomalía del uraco. 
paciente intervenido, correspondiente a un seno uracal, fue necesario realizar una incisión adicional periumbilical para extracción de la lesión, debido a su localización supraaponeurótica.

El tiempo quirúrgico promedio fue de 63 minutos (45, 110); este fue disminuyendo progresivamente a medida que se realizaban los casos sucesivos, alcanzándose una meseta a partir del quinto caso, en probable relación con la curva de aprendizaje. En una paciente se asoció al procedimiento, cura operatoria de hernia inguinal indirecta izquierda por técnica laparoscópica transperitoneral (Tabla 1).

Tabla 1. Variables demográficas y perioperatorios de los pacientes tratados

\begin{tabular}{lc}
\hline Variable & Promedio (Rango) \\
\hline Edad & $19(15-37)$ \\
Relación hombres: mujeres & $2: 12$ \\
Tiempo quirúrgico (minutos) & $63(45-110)$ \\
Estancia hospitalaria (horas) & $16(8-48)$ \\
Tiempo de seguimiento (meses) & $28(6-72)$ \\
\hline
\end{tabular}

La pérdida estimada de sangre fue mínima (menor de 50cc) y no se presentaron complicaciones intraoperatorias ni casos de conversión a cirugía abierta. Las complicaciones postoperatorias reportadas corresponden a dos casos de cistitis postoperatoria en relación con la cateterización de la vejiga, los cuales respondieron satisfactoriamente a tratamiento médico.

La paciente a quien se le realizó durante el mismo procedimiento cura operatoria de hernia inguinal indirecta por técnica laparoscópica transperitoneal, presentó sangrado postoperatorio por lesión de la vena epigástrica que ameritó transfusión y revisión laparoscópica de la cavidad a las 12 horas para hemostasia y lavado, con evolución postoperatoria favorable.

Cinco de los procedimiento (35\%) se realizaron de manera ambulatoria, el resto de los pacientes permanecieron hospitalizados por 24 horas, con excepción de la paciente que amerito reintervención por complicación relacionada con procedimiento asociado.

Del total de pacientes tratados se han controlado nueve de ellos, con un período de seguimiento promedio de 28 meses (6 - 72), hasta la fecha ninguno ha reportado recurrencia de la sintomatología y la única complicación tardía corresponde a una eventración sintomática en el portal número uno, aún no corregida.

\section{DISCUSIÓN}

El uraco es un remanente embriológico del alantoides, la cual hacia el quinto mes de gestación comienza a obliterarse y para el momento del nacimiento se encuentra totalmente ocluida dando origen al ligamento umbilical medio, que presenta variantes anatómicas descritas por Hammond et al. ${ }^{11}$, siendo la más frecuente la tipo III, es decir, la unión de este ligamento medio con ambas arteria umbilicales obliteradas para llegar al ombligo como un elemento único, tal y como se confirmó en el estudio.

Las anomalías del uraco no son frecuentes, se calcula c en 1 en 5000, la edad de presentación es muy variable y aunque no se ha reportado en la literatura preferencia por algún sexo, la gran mayoría de los pacientes en este estudio corresponden al sexo femenino.

La patología se presenta en casos en que el uraco no se oblitera totalmente luego del nacimiento (patología uracal congénita) o en casos en que ha cerrado normalmente pero sufre una reapertura parcial o total (patología uracal adquirida). En relación con esta repermeabilización Bauer y Retik ${ }^{12}$ describen cuatro variantes de las anomalías del uraco. En general el diagnóstico de anomalía del uraco es difícil debido a su baja incidencia, ausencia de signos y síntomas específicos y la posibilidad de simular otros cuadros abdominales.

El divertículo vesicouracal, o anomalía tipo I, se presenta como infecciones urinarias bajas a repetición $u$ otros síntomas urinarios inespecíficos que rara vez conducen a un diagnóstico.

El seno uracal, que parece ser la anomalía más frecuente ${ }^{5}$, se presenta como episodios de secreción umbilical clara o purulenta, acompañada o no de dolor abdominal periumbilical y fiebre. La descrita tríada clásica caracterizada por descarga umbilical de orina o secreción acuosa, dolor abdominal y síntomas urinarios tipo disuria o poliaquiuria ${ }^{4}$, se presenta sólo en casos de anomalías tipo III, es decir, uraco patente en todo su trayecto.

Si se trata de una formación quística, rara vez se hace un diagnóstico precoz, sin embargo, si este quiste se infecta lo cual ocurre generalmente por vía hematógena o posterior a traumatismos, se presenta como un cuadro de dolor abdominal, fiebre y en ocasiones masa palpable ${ }^{13}$. El germen involucrado es generalmente el Staphylococcus aureus de origen cutáneo, aunque se han aislado otros gérmenes 
como escherichia coli, proteus mirabilis, staphylococcus epidermidis, y hasta en un $36 \%$ de los casos la infección es polimicrobiana ${ }^{5}$.

Para el diagnóstico certero preoperatorio se pueden utilizar, según el tipo de anomalía uracal sospechada, los siguientes exámenes: 1. Sinograma o fistulografía, útil en los casos en que existe comunicación con el ombligo. La cateterización del trayecto y la inyección del contraste permite confirmar el diagnóstico y determinar el tipo de anomalía de uraco presente; 2. Ultrasonido abdominal; 3. Cistografía miccional; 4. Tomografía axial computarizada, debido a su baja disponibilidad y alto costo, se reserva para casos en lo que sospecha malignidad, sin embargo, en los casos con sintomatología inespecífica este estudio resulta de gran utilidad debido a que permite descartar la presencia de otras patologías abdominales.

A pesar de los estudios mencionados, en muchos casos el diagnóstico definitivo se establece durante la realización del procedimiento quirúrgico. En ocasiones el diagnóstico se realiza de manera incidental durante la realización de un procedimiento laparoscópico por otra patología, reportándose incluso la lesión inadvertida de quiste de uraco al momento de realizar el neumoperitoneo ${ }^{14}$.

El tratamiento aceptado involucra la resección del uraco en todo su trayecto, incluyendo el peritoneo parietal dejando únicamente los extremos umbilical y vesical, ya que la resecciones parciales se asocian a una recurrencia alrededor del 30\% ${ }^{7}$; los casos de infección pueden ser tratados en dos tiempos quirúrgicos.

En la literatura mundial se han reportado varios casos de degeneración maligna del uraco hacia adenocarcinomas, principalmente de tipo mucinoso, cáncer de células transicionales y cáncer epidermoide. La incidencia exacta de este tipo de cambios neoplásicos es desconocida, pero se calcula alrededor del 0,01\% $\%^{15}$. La presencia de una masa palpable en la línea media infraumbilical y hematuria en conjunto con el hallazgo de calcificaciones en estudios radiológicos, son altamente sugestivo de malignidad. Basados en este potencial de transformación maligna y el ya descrito trayecto intramural del uraco en su extremo vesical, algunos autores consideran que el tratamiento debe incluir una resección parcial de la vejiga ${ }^{8}$. Sin embargo, consideramos que las probabilidades de este tipo de degeneración son muy bajas para justificar la realización de cis- tectomía parcial, lo cual sin duda agrega morbilidad al procedimiento. Luego de la introducción de la cirugía laparoscópica este tipo de abordaje ha venido cobrando auge en el campo de la cirugía general y la urología, siempre aportando las ya conocidas bondades de la cirugía minimamente invasiva, es decir, menor dolor, menor tiempo de hospitalización, rápida reincorporación a las actividades habituales y un mucho mejor resultado estético.

La utilidad del abordaje laparoscópico para el tratamiento de las anomalías sintomáticas del uraco fue descrita por primera vez por Trondsen et al. ${ }^{9}$ posteriormente otros autores han descrito casos aislados tratados por esta vía, tal es el caso de Siegel et al. ${ }^{16}$, Fahlenkamp et al. ${ }^{17}$. Yamada et al. describieron la resección manoasistida de un quiste gigante de uraco $^{18}$. Así mismo, Cadeddu et al. ${ }^{19}$ en el año 2000 evalúan de manera retrospectiva una serie de cuatro casos, Khurana y Boris ${ }^{20}$ reportaron la utilidad del abordaje en el tratamiento de cuatro pacientes pediátricos también con excelentes resultados. Nakagawa ${ }^{21}$ reportó en el 2002 una serie de once pacientes con excelentes resultados y una lesión de la vejiga como una complicación asociada al procedimiento laparoscópico.

LePicard y Carrion describen una técnica endoscópica extraperitoneal ${ }^{22}$. Más recientemente en el 2005 Cutting et al. ${ }^{23}$ describieron su experiencia en el tratamiento mínimo invasivo de cinco pacientes, reportando un caso de recurrencia debido a anomalía de uraco supraaponeurótica en el extremo umbilical, no advertida.

Okegawa et al., tienen el mérito de haber publicado a la fecha, el único estudio comparativo entre la técnica laparoscópica y la cirugía convencional, reportando clara ventajas para la primera, en relación con estancia hospitalaria y reincorporación a las actividades habituales ${ }^{24}$.

El presente estudio representa hasta la fecha la serie más numerosa en reportada en la literatura que demuestra la factibilidad, seguridad y eficacia de la resección laparoscópica del uraco cuando están presentes anomalías sintomáticas, con un tiempo quirúrgico promedio aceptable, similar al reportado por otros autores, y por supuesto menor a cuando se realiza resecciones parciales de la vejiga. Reportándose sólo complicaciones menores (cistitis postoperatoria, eventración en portal) relacionadas con el procedimiento y sin recurrencia en el seguimiento a largo plazo. 
La técnica propuesta por los autores consiste en la utilización de tres portales de $10 \mathrm{~mm}$, lo cual permite la resección del uraco completamente, sin embargo, debe tenerse en cuenta que el abordaje laparoscópico no permite la resección de anomalías del uraco cuando se encuentran en su corto trayecto supraaponeurótico en el extremo umbilical, en cuyo caso es necesario realizar una incisión umbilical adicional para su escisión, y de esta manera evitar la recurrencia relacionada con esta técnica, debido a esta localización poco habitual similar a la reportada en la serie de Cutting et al. ${ }^{23}$. A pesar, de que se han descrito varios casos de degeneración maligna, hasta ahora en nuestra serie no se han encontrado cambios neoplásicos en ninguno de los especímenes.

Si bien es cierto que para evaluar una técnica novedosa lo ideal es realizar estudios prospectivos, aleatorios y controlados, la baja incidencia de las anomalías del uraco en la población en general representa una gran limitación para tal fin. Sin embargo, parece razonable en base a la evidencia actual, sugerir que la laparoscopia al ofrecer una vista panorámica tiene un gran papel en el diagnóstico y tratamiento de la patología del uraco, ofreciendo las ya mencionadas ventajas, además de probablemente menor morbilidad que la cirugía abierta y un óptimo resultado estético.

\section{CONCLUSIÓN}

La laparoscopia juega un importante papel en el manejo de las anomalías sintomáticas del uraco, ya que permite confirmar el diagnóstico clínico y realizar la resección del uraco de manera segura y eficaz, aportando la ya conocida ventaja de la cirugía mínimamente invasiva. Los estudios de evalución preoperatorio son de poca utilidad en la confirmación de esta patología.

\section{REFERENCIAS}

1. Berman S, Tolia B, Laor E, Reid R, Schweizerhof S, Freed S. Urachal remnants in adults. Urology 1988;31(1):17-21.

2. Blichert-Toft M, Koch F, Nielsen OV. Anatomic variants of the urachus related to clinical appearance and surgical treatment of urachal lesions. Surg Gynecol Obstect 1973;37(1):51-55.

3. Begg R. The urachus and umbilical fistulae. Surg Gynec Obstect 1927; 45:167-78

4. Sterling J, Goldsmith R. Lesions of the urachus which appear in the adult. Ann Surg 1953;137(1):120-128.
5. Cilento B, Bauer S, Retik A, Peters C, Atala A. Urachal anomalies: defining the best diagnostic modality. Pediatric Urology 1998;52(1):120-122

6. Rich RH, Hardy BE, Tiller RM. Surgery for anomalies of the urachus. J Ped Surg 1983;18(4):370-372.

7. Nix J, Menville J, Albert M, Wendt D. Congenital patent urachus. J Urol 1958;79(2):264-273.

8. Mesrobian H, Zacharias A, Balcom A, Cohen R. Ten years of experience with isolated urachal anomalies in children. J Urol 1997;158(3 Pt 2):1316-1318.

9. Trondsen E, Reiertsen O, Roseeland AR. Laparoscopic excision of a urachal sinus. Eur J Surg 1993;159:127-128 ABSTRACT.

10. Navarrete S, Sánchez-Ismayel A, Sánchez-Salas R, Sánchez R, Navarrete S. Treatment of urachal anomalies: a minimally invasive surgery technique. JSLS 2005;9(4):422-425

11. Hammond G, Yglesias L, Davis J. The urachus its anatomy and associated fascie. Anat Rec 1941;80:271-273.

12. Bauer SB, Retik AB. Urachal abnormalities and related umbilical disorders. Urol Clin Of North Am 1978;5(1):195-211.

13. Goldman IL, Caldamone A, Gauderer M, Hampel N, Wesselhoeft CW, et al. Infected urachal cyst: a review of 10 cases. J Urol 1988;140(2):375-378.

14. McLucas B, March C. Urachal sinus perforation during laparoscopy. A case report. J Reprod Med 1990;35(5):573-574.

15. Pantuck A, Vates T, Javidian P, Weiss R. Urachal adenocarcinoma. Can J Urol 1997;4(4):450-452.

16. Siegel JF, Winfield H, Valderrama E, Smith A. Laparoscopic excision of urachal cyst. J Urol 1994;151(6):1631-1633.

17. Fahlenkamp D, Schonberger B, Lindeke A, Loening S. Laparoscopic excision of the sinusoidal remmants of the urachus in a 3 year old boy. Br J Urol 1995;76(1):135-137.

18. Yamada T, Okamoto Y, Kasamatsu H, Mori H. Laparoscopic assisted removal of a large urachal cyst. J Am Assoc Gynecol Laparosc 2001;8(1):159-160.

19. Cadeddu JA, Boyle KE, Fabrizio MD, Chulam OG, Kavoussi LR. Laparoscopic management of urachal cysts in adulthood. J Urol 2000;164(5):1526-1528

20. Khurana S, Borzi PA. Laparoscopic management of complicated urachal disease in children. J Urol 2002;168(4 Pt 1):15261528.

21. Nakagawa K. Laparoscopic excision of urachal ramnant. JJCS 2002;27:884-888.

22. LePicard P, Carion G. Extraperitoneal laparoscopic treatment of urachal cyst. J Chir 2002;139(5):286-287.

23. Cutting CW, Hindley RG, Poulsen J. Laparoscopic management of complicates urachal remnants. BJU Int 2005;96(9):14171421.

24. Okegawa T, Odagane A, Nutahara K, Higashihara E. Laparoscopic management of urachal remnants in adulthood. Int $\mathrm{J}$ Urol 2006;13(12):1466-1469.

Correspondencia autor: Dr. Rafael E. Sánchez-Salas Servicio de Urología. Hospital Universitario de Caracas. Universidad Central de Venezuela

Caracas, Venezuela

Fax: 0582122658482

E-mail: raersas@hotmail.com

Información artículo: Original - Laparoscopia

Trabajo recibido: diciembre 2008

Trabajo aceptado: enero 2009 